\title{
KY FAN INEQUALITY AND BOUNDS FOR DIFFERENCES OF MEANS
}

\section{PENG GAO}

Received 23 July 2002

\begin{abstract}
We prove an equivalent relation between Ky Fan-type inequalities and certain bounds for the differences of means. We also generalize a result of Alzer et al. (2001).
\end{abstract}

2000 Mathematics Subject Classification: 26D15, 26D20.

1. Introduction. Let $P_{n, r}(\mathbf{x})$ be the generalized weighted power means: $P_{n, r}(\mathbf{x})=\left(\sum_{i=1}^{n} \omega_{i} x_{i}^{r}\right)^{1 / r}$, where $\omega_{i}>0,1 \leq i \leq n$ with $\sum_{i=1}^{n} \omega_{i}=1$ and $\mathbf{x}=$ $\left(x_{1}, x_{2}, \ldots, x_{n}\right)$. Here, $P_{n, 0}(\mathbf{x})=\prod_{i=1}^{n} x_{i}^{\omega_{i}}$ denotes the limit of $P_{n, r}(\mathbf{x})$ as $r \rightarrow 0^{+}$, which can be proved by noting that if $p(r)=\ln \left(\sum_{i=1}^{n} \omega_{i} x_{i}^{r}\right)$, then $p^{\prime}(0)=$ $\ln \left(\prod_{i=1}^{n} x_{i}^{\omega_{i}}\right)=\ln \left(P_{n, 0}(\mathbf{x})\right)$. We write $P_{n, r}$ for $P_{n, r}(\mathbf{x})$ when there is no risk of confusion.

In this paper, we assume that $0<x_{1} \leq x_{2} \leq \cdots \leq x_{n}$. With any given $\mathbf{x}$, we associate $\mathbf{x}^{\prime}=\left(1-x_{1}, 1-x_{2}, \ldots, 1-x_{n}\right)$ and write $A_{n}=P_{n, 1}, G_{n}=P_{n, 0}$, and $H_{n}=P_{n,-1}$. When $1-x_{i} \geq 0$ for all $i$, we define $A_{n}^{\prime}=P_{n, 1}\left(\mathbf{x}^{\prime}\right)$ and similarly for $G_{n}^{\prime}$ and $H_{n}^{\prime}$. We also let $\sigma_{n}=\sum_{i=1}^{n} \omega_{i}\left[x_{i}-A_{n}\right]^{2}$.

The following counterpart of the arithmetic mean-geometric mean inequality, due to Ky Fan, was first published by Beckenbach and Bellman [7].

THEOREM 1.1. For $x_{i} \in(0,1 / 2]$,

$$
\frac{A_{n}^{\prime}}{G_{n}^{\prime}} \leq \frac{A_{n}}{G_{n}}
$$

with equality holding if and only if $x_{1}=\cdots=x_{n}$.

In this paper, we consider the validity of the following additive Ky Fan-type inequalities (with $x_{1}<x_{n}<1$ ):

$$
\frac{x_{1}}{1-x_{1}}<\frac{P_{n, r}^{\prime}-P_{n, s}^{\prime}}{P_{n, r}-P_{n, s}}<\frac{x_{n}}{1-x_{n}}
$$

Note that by a change of variables $x_{i} \rightarrow 1-x_{i}$, the left-hand side inequality is equivalent to the right-hand side inequality in (1.2). We can deduce (see [9]) Theorem 1.1 from the case $r=1, s=0$, and $x_{n} \leq 1 / 2$ in (1.2), which is a result 
of Alzer [5]. Gao [9] later proved the validity of (1.2) for $r=1,-1 \leq s<1$, and $x_{n} \leq 1 / 2$.

What is worth mentioning is a nice result of Mercer [12] who showed that the validity of $r=1$ and $s=0$ in (1.2) is a consequence of a result of Cartwright and Field [8] who established the validity of $r=1$ and $s=0$ for the following bounds for the differences between power means $(r>s)$ :

$$
\frac{r-s}{2 x_{1}} \sigma_{n} \geq P_{n, r}-P_{n, s} \geq \frac{r-s}{2 x_{n}} \sigma_{n}
$$

where the constant $(r-s) / 2$ is the best possible (see [10]).

We point out that inequalities (1.2) and (1.3) do not hold for all $r>s$. We refer the reader to the survey article [2] and the references therein for an account of Ky Fan's inequality, and to $[4,5,10,11]$ for other interesting refinements and extensions of (1.3).

Mercer's result reveals a close relation between (1.3) and (1.2), and it is our main goal in the paper to prove that the validities of (1.3) and (1.2) are equivalent for fixed $r$ and $s$. As a consequence of this result, we give a characterization of the validity of (1.3) for $r=1$ or $s=1$. A solution of an open problem from [11] is also given.

Among the numerous sharpenings of Ky Fan's inequality in the literature, we have the following inequalities connecting the three classical means (with $\omega_{i}=1 / n$ here):

$$
\left(\frac{H_{n}}{H_{n}^{\prime}}\right)^{n-1} \frac{A_{n}}{A_{n}^{\prime}} \leq\left(\frac{G_{n}}{G_{n}^{\prime}}\right)^{n} \leq\left(\frac{A_{n}}{A_{n}^{\prime}}\right)^{n-1} \frac{H_{n}}{H_{n}^{\prime}} .
$$

The right-hand side inequality of (1.4) is due to W. L. Wang and P. F. Wang [14] and the left-hand side inequality was recently proved by Alzer et al. [6].

It is natural to ask whether we can extend the above inequality to the weighted case, and using the same idea as in [6], we show that this is indeed true in Section 5.

\section{The main theorem}

THEOREM 2.1. For fixed $r>s$, the following inequalities are equivalent: (i) inequality (1.2) for $x_{n} \leq 1 / 2$; (ii) inequality (1.2); (iii) inequality (1.3).

Proof. (iii) $\Rightarrow$ (ii) follows from a similar argument as given in [12], (ii) $\Rightarrow$ (i) is trivial, so it suffices to show that (i) $\Rightarrow$ (iii).

Fix $r>s$ assuming that (1.2) holds for $x_{n} \leq 1 / 2$. Without loss of generality, we can assume that $x_{1}<x_{n}$. For a given $\mathbf{x}=\left(x_{1}, x_{2}, \ldots, x_{n}\right)$, let $\mathbf{y}=$ $\left(\epsilon X_{1}, \epsilon X_{2}, \ldots, \epsilon x_{n}\right)$. We can choose $\epsilon$ small so that $\epsilon x_{n} \leq 1 / 2$. Now, applying the right-hand side inequality (1.2) for $\mathbf{y}$, we get

$$
x_{n}\left(P_{n, r}(\mathbf{x})-P_{n, s}(\mathbf{x})\right)>\frac{1-\epsilon x_{n}}{\epsilon^{2}}\left(P_{n, r}\left(\mathbf{y}^{\prime}\right)-P_{n, s}\left(\mathbf{y}^{\prime}\right)\right) .
$$


Let $f(\epsilon)=P_{n, r}\left(\mathbf{y}^{\prime}\right)-P_{n, s}\left(\mathbf{y}^{\prime}\right)$, then $f^{\prime}(0)=0$ and $f^{\prime \prime}(0)=(r-s) \sigma_{n}$. Thus, by letting $\epsilon$ tend to 0 , it is easy to verify that the limit of the expression on the right-hand side of (2.1) is $(r-s) \sigma_{n} / 2$. We can consider the left-hand side of (1.2) by a similar argument and this completes the proof.

\section{An application of Theorem 2.1}

LEMMA 3.1. If inequality (1.3) holds for $r>s$, then $0 \leq r+s \leq 3$.

Proof. Let $n=2$, and write $\omega_{1}=1-q, \omega_{2}=q, x_{1}=1$, and $x_{2}=1+t$ with $t \geq-1$. Let

$$
D(t ; r, s, q)=\frac{r-s}{2} \sum_{i=1}^{2} w_{i}\left[x_{i}-A_{2}\right]^{2}-P_{2, r}+P_{2, s}
$$

For $t \geq 0, D(t ; r, s, q) \geq 0$ implies the validity of the left-hand side inequality of (1.3) while for $-1 \leq t \leq 0, D(t ; r, s, q) \leq 0$ implies the validity of the righthand side inequality of (1.3).

Using the Taylor series expansion of $D(t ; r, s, q)$ around $t=0$, it is readily seen that $D(0 ; r, s, q)=D^{(1)}(0 ; r, s, q)=D^{(2)}(0 ; r, s, q)=0$. Thus, by the Lagrangian remainder term of the Taylor expansion,

$$
D(t ; r, s, q)=\frac{D^{(3)}(\theta t ; r, s, q)}{3 !} t^{3}
$$

with $0<\theta<1$.

Since

$$
\lim _{t \rightarrow 0^{+}} D^{(3)}(\theta t ; r, s, q)=D^{(3)}(0 ; r, s, q),
$$

a necessary condition for (1.3) to hold is $D^{(3)}(0 ; r, s, q) \geq 0$ for $0 \leq q \leq 1$. The calculation yields

$$
D^{(3)}(0 ; r, s, q)=(r-s) q(q-1)((3-2 r-2 s) q-(3-r-s))
$$

It is easy to check that this is equivalent to $0 \leq r+s \leq 3$.

THEOREM 3.2. Let $r>s$. If $r=1$, inequality (1.3) holds if and only if $-1 \leq$ $s<1$. If $s=1$, inequality (1.3) holds if and only if $1<r \leq 2$.

Proof. A result of Gao [9] shows the validity of (1.2) for $r=1,-1 \leq s<1$, $x_{n} \leq 1 / 2$, and a similar result of his [10] shows the validity of (1.2) for $s=1$, $1<r \leq 2, x_{n} \leq 1 / 2$. Thus, it follows from Theorem 2.1 that (1.3) holds for $r=$ $1,-1 \leq s<1$, and $s=1,1<r \leq 2$. This proves the "if" part of the statement, and the "only if" part follows from the previous lemma. 
We note here that a special case of Theorem 3.2 answers an open problem of Mercer [11], namely, we have shown that

$$
\frac{1}{x_{1}} \sigma_{n} \geq A_{n}-H_{n} \geq \frac{1}{x_{n}} \sigma_{n}
$$

\section{Two lemmas}

LEMMA 4.1. Let $x, b, u$, and $v$ be real numbers with $0<x \leq b, u \geq 1, v \geq 0$, and $u+v \geq 2$, then $f(u, v, x, b) \leq 0$, where

$$
f(u, v, x, b)=\frac{u+v-1}{u x+v b}+\frac{1}{x^{2}(u / x+v / b)}-\frac{1}{x}-\frac{u+v-2}{b^{2}(u+v)^{2}} v(x-b)
$$

with equality holding if and only if $x=b$ or $v=0$ or $u=v=1$.

Proof. Let $x<b, u>1$, and $v>1$. We have

$$
\begin{aligned}
f(u, v, x, b) & =v(b-x)\left(-\frac{(u-1) b+(v-1) x}{x(b v+u x)(b u+v x)}+\frac{(u-1)+(v-1)}{b^{2}(u+v)^{2}}\right) \\
& <\frac{v(b-x)}{x b^{2}(u+v)^{2}}[((u-1)+(v-1)) x-(u-1) b-(v-1) x] \\
& =-\frac{v(u-1)(b-x)^{2}}{x b^{2}(u+v)^{2}}<0
\end{aligned}
$$

since $b^{2}(u+v)^{2}>(b v+u x)(b u+v x)$. Thus, we conclude that $f(u, v, x, b) \leq$ 0 for $0<x \leq b, u \geq 1, v \geq 0$, and $u+v \geq 2$.

LEMMA 4.2. Let $x, a, b, u, v$, and $s$ be real numbers with $0<x \leq a \leq b$, $u \geq 1, v \geq 1, u+v \geq 3$, and $0 \leq s \leq v$, then

$$
\begin{gathered}
\frac{u+v-1}{u x+s a+(v-s) b}+\frac{1}{x^{2}(u / x+s / a+(v-s) / b)}-\frac{1}{x} \\
-\frac{u+v-2}{b^{2}(u+v)^{2}}(s(x-a)+(v-s)(x-b)) \leq 0
\end{gathered}
$$

with equality holding if and only if one of the following cases is true: (1) $x=a=$ $b$; (2) $s=0$ and $x=b$; (3) $s=v$ and $x=a$.

Proof. Let $M=\left\{(s, a) \in R^{2} \mid 0 \leq s \leq v, x \leq a \leq b\right\}$. Furthermore, we define $H(s, a)$ as the expression on the left-hand side of $(4.3)$, where $(s, a) \in M$. It suffices to show that $H(s, a)<0$. We denote the absolute minimum of $H$ by $m=\left(s_{0}, a_{0}\right)$. If $m$ is an interior point of $M$, then we obtain

$$
0=\frac{1}{s} \frac{\partial H}{\partial a}-\left.\frac{1}{a-b} \frac{\partial H}{\partial s}\right|_{(s, a)=\left(s_{0}, a_{0}\right)}=\frac{b-a}{x^{4} a^{2} b(u / x+s / a+(v-s) / b)^{2}}>0 .
$$


Hence, $m$ is a boundary point of $M$, so we get

$$
m \in\left\{\left(s_{0}, x\right),\left(s_{0}, b\right),\left(0, a_{0}\right),\left(v, a_{0}\right)\right\} .
$$

Using Lemma 4.1, we obtain

$$
\begin{aligned}
& H\left(s_{0}, x\right)=f\left(u+s_{0}, v-s_{0}, x, b\right) \leq 0 \\
& H\left(s_{0}, b\right)=H\left(0, a_{0}\right)=f(u, v, x, b) \leq 0 \\
& H\left(v, a_{0}\right)=f\left(u, v, x, a_{0}\right)-\frac{v(u+v-2)\left(a_{0}-x\right)\left(b^{2}-a_{0}^{2}\right)}{a_{0}^{2} b^{2}(u+v)^{2}} \leq 0 .
\end{aligned}
$$

Thus, we get that if $(s, a) \in M$, then $H(s, a) \leq 0$. The conditions for equality can be easily checked using Lemma 4.1 .

5. A sharpening of Ky Fan's inequality. In this section, we prove the following theorem.

THEOREM 5.1. For $0<x_{1} \leq \cdots \leq x_{n}, q=\min \left\{\omega_{i}\right\}$,

$$
\begin{aligned}
& \frac{1-2 q}{2 x_{1}^{2}} \sigma_{n} \geq(1-q) \ln A_{n}+q \ln H_{n}-\ln G_{n} \geq \frac{1-2 q}{2 x_{n}^{2}} \sigma_{n}, \\
& \frac{1-2 q}{2 x_{1}^{2}} \sigma_{n} \geq \ln G_{n}-q \ln A_{n}-(1-q) \ln H_{n} \geq \frac{1-2 q}{2 x_{n}^{2}} \sigma_{n}
\end{aligned}
$$

with equality holding if and only if $q=1 / 2$ or $x_{1}=\cdots=x_{n}$.

Proof. The proof uses the ideas in [6]. We prove the right-hand side inequality of (5.1); the proofs for other inequalities are similar. Fix $0<x=x_{1}$, $x_{n}=b$ with $x_{1}<x_{n}, n \geq 2$; we define

$$
f_{n}\left(\mathbf{x}_{n}, q\right)=(1-q) \ln A_{n}+q \ln H_{n}-\ln G_{n}-\frac{1-2 q}{2 x_{n}^{2}} \sigma_{n},
$$

where we regard $A_{n}, G_{n}$, and $H_{n}$ as functions of $\mathbf{x}_{n}=\left(x_{1}, \ldots, x_{n}\right)$.

We then have

$$
g_{n}\left(x_{2}, \ldots, x_{n-1}\right):=\frac{1}{\omega_{1}} \frac{\partial f_{n}}{\partial x_{1}}=\frac{1-q}{A_{n}}+\frac{q H_{n}}{x_{1}^{2}}-\frac{1}{x_{1}}-\frac{1-2 q}{x_{n}^{2}}\left(x_{1}-A_{n}\right) .
$$

We want to show that $g_{n} \leq 0$. Let $D=\left\{\left(x_{2}, \ldots, x_{n-1}\right) \in R^{n-2} \mid 0<x \leq x_{2} \leq\right.$ $\left.\cdots \leq x_{n-1} \leq \boldsymbol{b}\right\}$. Let $\mathbf{a}=\left(a_{2}, \ldots, a_{n-1}\right) \in D$ be the point in which the absolute minimum of $g_{n}$ is reached. Next, we show that

$$
\mathbf{a}=(x, \ldots, x, a, \ldots, a, b, \ldots, b) \text { with } x<a<b,
$$

where the numbers $x, a$, and $b$ appear $r, s$, and $t$ times, respectively, with $r, s, t \geq 0$ and $r+s+t=n-2$. 
Suppose not, this implies that two components of a have different values and are interior points of $D$. We denote these values by $a_{k}$ and $a_{l}$. Partial differentiation leads to

$$
\frac{B}{a_{i}^{2}}+C=0
$$

for $i=k, l$, where

$$
B=q \frac{H_{n}^{2}}{x_{1}^{2}}, \quad C=-\frac{1-q}{A_{n}^{2}}+\frac{1-2 q}{x_{n}^{2}} .
$$

Since $z \mapsto B / z^{2}+C$ is strictly monotonic for $z>0$, then (5.6) yields $a_{k}=a_{l}$. This contradicts our assumption that $a_{k} \neq a_{l}$. Thus, (5.5) is valid and it suffices to show that $g_{n} \leq 0$ for the case $n=2,3$.

When $n=2$, by setting $x_{1}=x, x_{2}=b, \omega_{1} / q=u$, and $\omega_{2} / q=v$, we can identify $g_{2}$ as (4.1), and the result follows from Lemma 4.1.

When $n=3$, by setting $x_{1}=x, x_{2}=a, x_{3}=b, \omega_{1} / q=u, \omega_{2} / q=s$, and $\omega_{3} / q=v-s$, we can identify $g_{3}$ as (4.3), and the result follows from Lemma 4.2 .

Thus, we have shown that $g_{n}=\left(1 / \omega_{1}\right) \partial f_{n} / \partial x_{1} \leq 0$ with equality holding if and only if $n=1$ or $n=2, q=1 / 2$. By letting $x_{1}$ tend to $x_{2}$, we have

$$
f_{n}\left(\mathbf{x}_{n}, q\right) \geq f_{n-1}\left(\mathbf{x}_{n-1}, q\right) \geq f_{n-1}\left(\mathbf{x}_{n-1}, q^{\prime}\right),
$$

where $\mathbf{x}_{n-1}=\left(x_{2}, \ldots, x_{n}\right)$ with weights $\omega_{1}+\omega_{2}, \ldots, \omega_{n-1}, \omega_{n}$ and $q^{\prime}=\min \left\{\omega_{1}\right.$ $\left.+\omega_{2}, \ldots, \omega_{n}\right\}$. Here, we have used the following inequality, which is a consequence of (3.5) (see [9]):

$$
\ln A_{n}-\ln H_{n} \geq \frac{1}{x_{n}^{2}} \sigma_{n}
$$

It then follows by induction that $f_{n} \geq f_{n-1} \geq \cdots \geq f_{2}=0$ when $q=1 / 2$ in $f_{2}$ or else $f_{n} \geq f_{n-1} \geq \cdots \geq f_{1}=0$, and this completes the proof.

We note that the above theorem gives a sharpening of Sierpiński's inequality [13], originally stated for the unweighted case $\left(\omega_{i}=1 / n\right)$ as

$$
H_{n}^{n-1} A_{n} \leq G_{n} \leq A_{n}^{n-1} H_{n} .
$$

The following corollary gives refinements of (1.4). 
COROLLARY 5.2. For $0<x_{1} \leq \cdots \leq x_{n}<1, q=\min \left\{\omega_{i}\right\}$,

$$
\begin{aligned}
& \left(\frac{A_{n}^{\prime(1-q)} H_{n}^{\prime q}}{G_{n}^{\prime}}\right)^{\left(1-x_{1}\right)^{2} / x_{1}^{2}} \geq \frac{A_{n}^{1-q} H_{n}^{q}}{G_{n}} \geq\left(\frac{A_{n}^{\prime(1-q)} H_{n}^{\prime q}}{G_{n}^{\prime}}\right)^{\left(1-x_{n}\right)^{2} / x_{n}^{2}}, \\
& \left(\frac{G_{n}^{\prime}}{A_{n}^{\prime q} H_{n}^{\prime(1-q)}}\right)^{\left(1-x_{1}\right)^{2} / x_{1}^{2}} \geq \frac{G_{n}}{A_{n}^{q} H_{n}^{1-q}} \geq\left(\frac{G_{n}^{\prime}}{A_{n}^{\prime q} H_{n}^{\prime(1-q)}}\right)^{\left(1-x_{n}\right)^{2} / x_{n}^{2}},
\end{aligned}
$$

with equality holding if and only if $x_{1}=x_{2}=\cdots=x_{n}$ or $q=1 / 2$.

Proof. This is a direct consequence of Theorem 5.1, following from a similar argument as in [12].

6. Concluding remarks. We note that if for $x_{n} \leq 1 / 2$, we have

$$
\left(\frac{x_{1}}{1-x_{1}}\right)^{\beta}<\frac{P_{n, r}^{\prime}-P_{n, s}^{\prime}}{P_{n, r}-P_{n, s}}<\left(\frac{x_{n}}{1-x_{n}}\right)^{\alpha}
$$

then $\beta \geq 1$ and $\alpha \leq 1$; otherwise, by letting $\epsilon$ tend to 0 in (2.1), we get contradictions.

It was conjectured that an additive companion of (1.4) is true (see [1])

$$
n\left(G_{n}-G_{n}^{\prime}\right) \leq(n-1)\left(A_{n}-A_{n}^{\prime}\right)+H_{n}-H_{n}^{\prime} .
$$

In [3], Alzer asked if the above conjecture is true and whether there exists a weighted version. Based on what we have got in this paper, it is natural to give the following conjecture of the weighed version of (6.2).

CONJeCTURe 6.1. For $0<x_{1} \leq \cdots \leq x_{n} \leq 1 / 2$ and $q=\min \left\{\omega_{i}\right\}$,

$$
G_{n}-G_{n}^{\prime} \leq(1-q)\left(A_{n}-A_{n}^{\prime}\right)+q\left(H_{n}-H_{n}^{\prime}\right)
$$

Recently, Alzer et al. [6] asked the following question: what is the largest number $\alpha=\alpha(n)$ and what is the smallest number $\beta=\beta(n)$ such that

$$
\alpha\left(A_{n}-A_{n}^{\prime}\right)+(1-\alpha)\left(H_{n}-H_{n}^{\prime}\right) \leq G_{n}-G_{n}^{\prime} \leq \beta\left(A_{n}-A_{n}^{\prime}\right)+(1-\beta)\left(H_{n}-H_{n}^{\prime}\right)
$$

for all $x_{i} \in(0,1 / 2](i=1, \ldots, n)$ ?

We note here that $\alpha \leq 0$ since the left-hand side inequality above can be written as

$$
\alpha A_{n}+(1-\alpha) H_{n}-G_{n} \leq \alpha A_{n}^{\prime}+(1-\alpha) H_{n}^{\prime}-G_{n}^{\prime}
$$


By a similar argument as in the proof of Theorem 2.1, replacing $\left(x_{1}, \ldots, x_{n}\right)$ by $\left(\epsilon X_{1}, \ldots, \epsilon X_{n}\right)$ and letting $\epsilon$ tend to 0 in (6.5), we find that (6.5) implies that

$$
\alpha A_{n}+(1-\alpha) H_{n}-G_{n} \leq 0
$$

for any $\mathbf{x}$. If we further let $x_{1}$ tend to 0 in (6.6), we get

$$
\alpha A_{n} \leq 0
$$

which implies that $\alpha \leq 0$.

ACKNOWLEDGMENT. The author is grateful to the referees for their helpful comments and suggestions.

\section{REFERENCES}

[1] H. Alzer, An inequality for arithmetic and harmonic means, Aequationes Math. 46 (1993), no. 3, 257-263.

[2] _ The inequality of Ky Fan and related results, Acta Appl. Math. 38 (1995), no. 3, 305-354.

[3] _ On Ky Fan's inequality and its additive analogue, J. Math. Anal. Appl. 204 (1996), no. 1, 291-297.

[4] _ A new refinement of the arithmetic mean-geometric mean inequality, Rocky Mountain J. Math. 27 (1997), no. 3, 663-667.

[5] _ On an additive analogue of Ky Fan's inequality, Indag. Math. (N.S.) 8 (1997), no. 1, 1-6.

[6] H. Alzer, S. Ruscheweyh, and L. Salinas, On Ky Fan-type inequalities, Aequationes Math. 62 (2001), no. 3, 310-320.

[7] E. F. Beckenbach and R. Bellman, Inequalities, Ergebnisse der Mathematik und ihrer Grenzgebiete, N. F., Bd. 30, Springer-Verlag, Berlin, 1961.

[8] D. I. Cartwright and M. J. Field, A refinement of the arithmetic mean-geometric mean inequality, Proc. Amer. Math. Soc. 71 (1978), no. 1, 36-38.

[9] P. Gao, A generalization of Ky Fan's inequality, Int. J. Math. Math. Sci. 28 (2001), no. 7, 419-425.

[10] _ Certain bounds for the differences of means, RGMIA Res. Rep. Coll. 5 (2002), no. 3, Article 7.

[11] A. McD. Mercer, Bounds for $A-G, A-H, G-H$, and a family of inequalities of $K y$ Fan's type, using a general method, J. Math. Anal. Appl. 243 (2000), no. 1, 163-173.

[12] P. R. Mercer, A note on Alzer's refinement of an additive Ky Fan inequality, Math. Inequal. Appl. 3 (2000), no. 1, 147-148.

[13] W. Sierpiński, On an inequality for arithmetic, geometric and harmonic means, Warsch. Sitzungsber. 2 (1909), 354-358 (Polish).

[14] W. L. Wang and P. F. Wang, A class of inequalities for the symmetric functions, Acta Math. Sinica 27 (1984), no. 4, 485-497 (Chinese).

Peng Gao: Department of Mathematics, University of Michigan, Ann Arbor, MI 48109, USA

E-mail address: penggao@umich.edu 


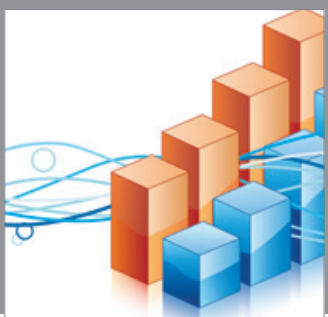

Advances in

Operations Research

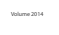

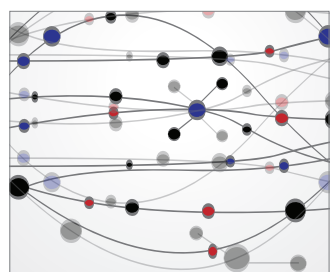

\section{The Scientific} World Journal
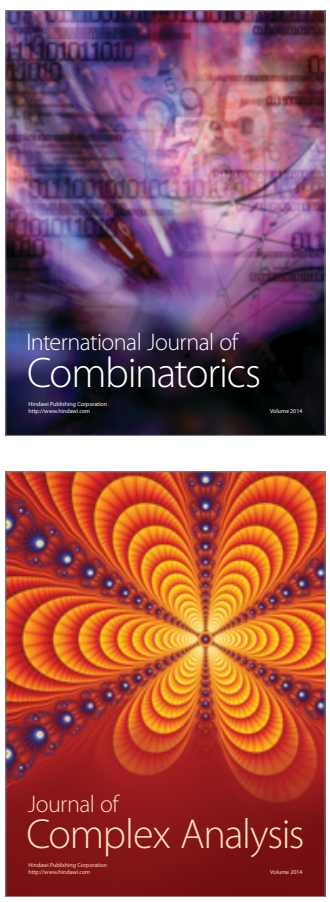

International Journal of

Mathematics and

Mathematical

Sciences
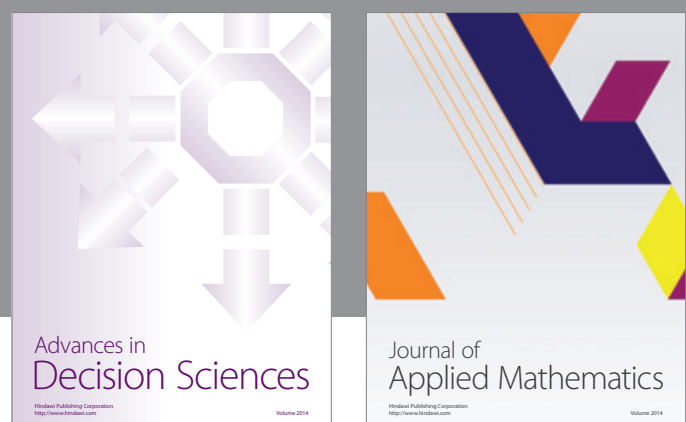

Journal of

Applied Mathematics
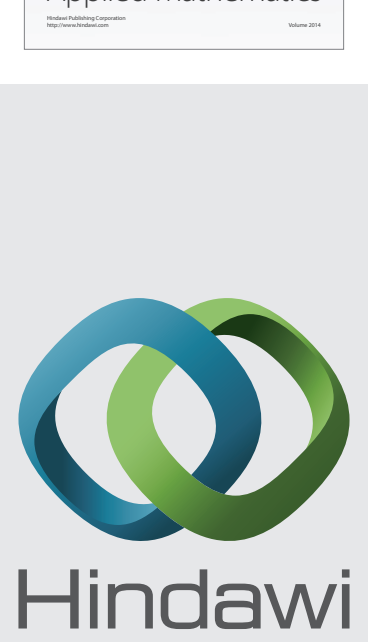

Submit your manuscripts at http://www.hindawi.com
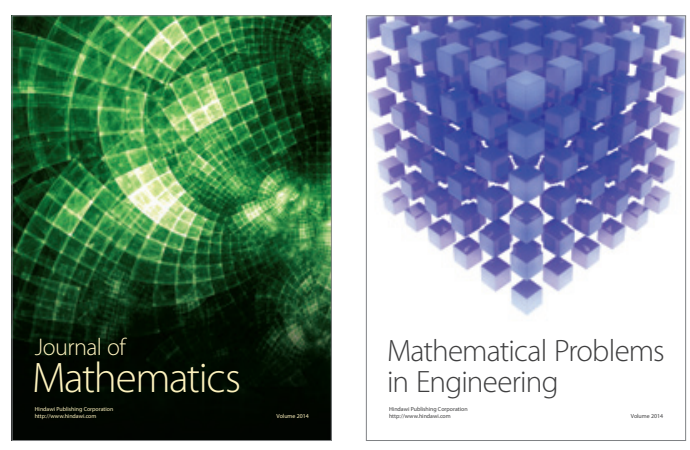

Mathematical Problems in Engineering
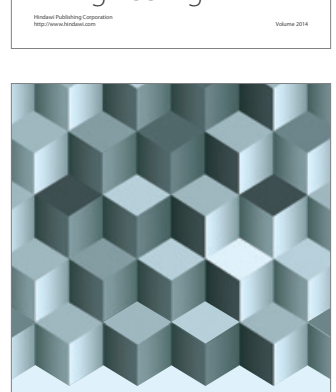

Journal of

Function Spaces
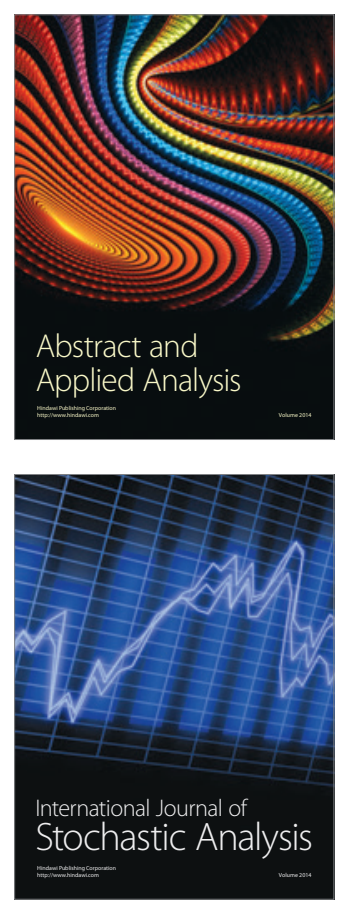

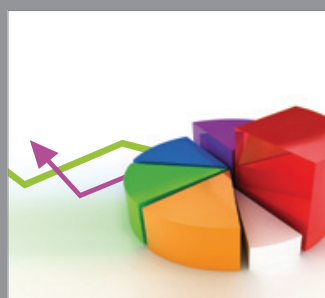

ournal of

Probability and Statistics

Promensencen
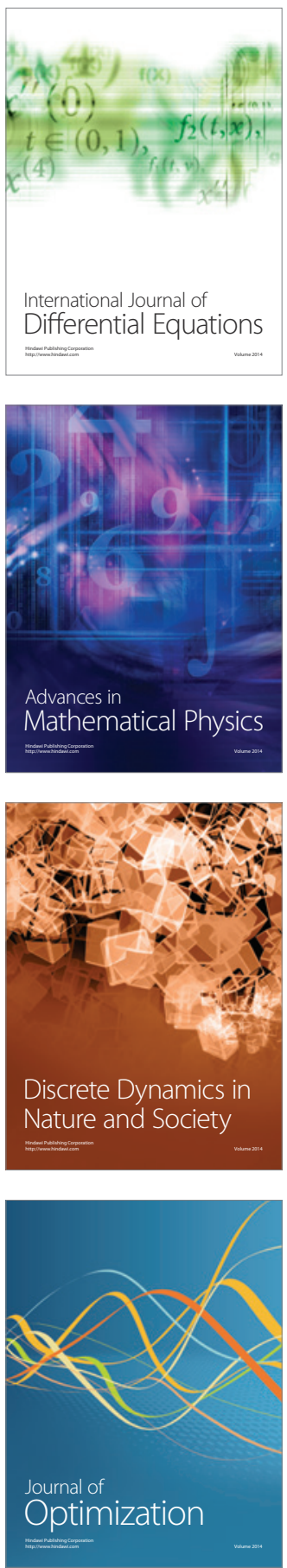